\title{
Probing Neuronal Nuclear Pore Complexes Using Single Molecule Localization Microscopy
}

\author{
Sumin Kim ${ }^{1}$, Sami Barmada ${ }^{2}$, William Dauer $^{3}$ and Sarah Veatch ${ }^{1}$ \\ ${ }^{1}$ University of Michigan, Ann Arbor, Michigan, United States, ${ }^{2}$ University of Michigan Medical School, \\ Ann Arbor, Michigan, United States, ${ }^{3}$ UT Southwestern Medical Center, Dallas, Texas, United States
}

Nuclear pore complexes (NPCs) are large protein complexes composed of hundreds of nucleoporins that mediate nucleocytoplasmic transport of protein and RNA ${ }^{1,2}$. Despite increasing evidence implicating NPC dysfunction in neurological diseases ${ }^{3-5}$, NPC biogenesis and function in neurons remain poorly understood. This is largely because most studies have been conducted in mitotic cells, in which NPC assembly is mechanistically distinct from interphase assembly occurring in neurons. NPC abnormalities are implicated in DYT1 dystonia ${ }^{6}$, a neurodevelopmental movement disorder caused by a loss-of-function mutation in torsinA, a AAA+ protein localized to the endoplasmic reticular (ER)/nuclear envelope (NE) endomembrane space ${ }^{7,8}$. How torsinA impacts NPC structure and function, and how loss of torsinA function ultimately cause DYT1 dystonia, remain unknown. In mouse primary neurons, we find a dramatic upregulation in nuclear pore complex biogenesis during neuronal maturation. In contrast to wild-type neurons, torsinA-null neurons develop increasingly mislocalized clusters of NPCs. Because traditional, diffraction-limited microscopy is insufficient to resolve the unique arrangement of NPCs, we used single molecule localization microscopy (SMLM) to elucidate the nature of mislocalized NPC clusters. The added resolution enabled us to observe that abnormal clusters of NPCs in torsinA-null neurons comprise structures with a central pore. This observation indicates that the clusters represent intermediate states of NPC assembly rather than aggregates of nucleoporins. This finding is in agreement with our previous observations that abnormal NPC clusters contain early- but not late-recruited NPC components. Together, our SMLM results support a model in which torsinA function is required for NPC assembly and organization in developing neurons, potentially impacting nucleocytoplasmic transport and contributing to DYT1 dystonia. Further, these observations hint at a critical period of accelerated NPC assembly during neuronal development that is essential for maintenance of neuronal function.

\section{References}

1. Kim SJ, Fernandez-Martinez J, Nudelman I, et al. Integrative structure and functional anatomy of a nuclear pore complex. Nature. 2018. doi:10.1038/nature26003

2. Knockenhauer KE, Schwartz TU. The Nuclear Pore Complex as a Flexible and Dynamic Gate. Cell. 2016;164(6):1162-1171. doi:10.1016/j.cell.2016.01.034

3. Grima JC, Daigle JG, Arbez N, et al. Mutant Huntingtin Disrupts the Nuclear Pore Complex. Neuron. 2017. doi:10.1016/j.neuron.2017.03.023

4. Chou C-C, Zhang Y, Umoh ME, et al. TDP-43 pathology disrupts nuclear pore complexes and nucleocytoplasmic transport in ALS/FTD. Nat Neurosci. 2018. doi:10.1038/s41593-017-0047-3

5. Eftekharzadeh B, Daigle JG, Kapinos LE, et al. Tau Protein Disrupts Nucleocytoplasmic Transport in Alzheimer's Disease. Neuron. 2018. doi:10.1016/j.neuron.2018.07.039

6. Pappas SS, Liang CC, Kim S, Rivera CAO, Dauer WT. TorsinA dysfunction causes persistent neuronal nuclear pore defects. Hum Mol Genet. 2018;27(3):407-420. doi:10.1093/hmg/ddx405 
7. Ozelius LJ, Hewett J, Kramer P, et al. Fine localization of the torsion dystonia gene (DYT1) on human chromosome 9q34: YAC map and linkage disequilibrium. Genome Res. 1997;7(5):483-494. doi:10.1101/gr.7.5.483

8. Goodchild RE, Kim CE, Dauer WT. Loss of the dystonia-associated protein torsinA selectively disrupts the neuronal nuclear envelope. Neuron. 2005;48(6):923-932. doi:10.1016/j.neuron.2005.11.010 